\title{
Article \\ Body Fat Distribution and Risk of Breast, Endometrial, and Ovarian Cancer: A Two-Sample Mendelian Randomization Study
}

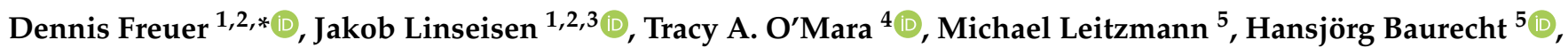 \\ Sebastian-Edgar Baumeister ${ }^{6}(1)$ and Christa Meisinger ${ }^{1}$
}

1 Chair of Epidemiology, University of Augsburg, University Hospital Augsburg, 86156 Augsburg, Germany; jakob.linseisen@med.uni-augsburg.de (J.L.); christine.meisinger@med.uni-augsburg.de (C.M.)

2 Institute for Medical Information Processing, Biometry, and Epidemiology, Ludwig-Maximilians-Universität München, 81377 Munich, Germany

3 German Research Center for Environmental Health, Independent Research Group Clinical Epidemiology, Helmholtz Zentrum München, 85764 Neuherberg, Germany

4 Genetics and Computational Biology Department, QIMR Berghofer Medical Research Institute, Brisbane 4006, Australia; Tracy.OMara@qimrberghofer.edu.au

5 Department of Epidemiology and Preventive Medicine, University of Regensburg, 93053 Regensburg, Germany; michael.leitzmann@klinik.uni-regensburg.de (M.L.); hansjoerg.baurecht@klinik.uni-regensburg.de (H.B.)

6 Institute of Health Services Research in Dentistry, University of Münster, 48149 Münster, Germany; sebastian.baumeister@uni-muenster.de

check for
updates

Citation: Freuer, D.; Linseisen, J.; O'Mara, T.A.; Leitzmann, M.; Baurecht, H.; Baumeister, S.-E.; Meisinger, C. Body Fat Distribution and Risk of Breast, Endometrial, and Ovarian Cancer: A Two-Sample Mendelian Randomization Study. Cancers 2021, 13, 5053. https:// doi.org/10.3390/cancers13205053

Academic Editors: Elaine F. Harkness and Andrea Manni

Received: 5 August 2021

Accepted: 6 October 2021

Published: 9 October 2021

Publisher's Note: MDPI stays neutral with regard to jurisdictional claims in published maps and institutional affiliations.

Copyright: (c) 2021 by the authors. Licensee MDPI, Basel, Switzerland. This article is an open access article distributed under the terms and conditions of the Creative Commons Attribution (CC BY) license (https:/ / creativecommons.org/licenses/by/ $4.0 /)$.
* Correspondence: dennis.freuer@med.uni-augsburg.de; Tel.: +49-821-598-6474

Simple Summary: The causal impact of body fat distribution on female-specific cancers is largely unknown. For the first time we used a two-sample multivariable Mendelian randomization (MR) approach to elucidate the role and causal relations of body composition assessed by segmental bioelectrical impedance analysis on the risks of breast, endometrial and ovarian cancers and their subtypes. We found that abdominal fat content increases the risk for ovarian cancer and its endometrioid and clear cell subtypes independent of overall fat content. General adiposity has a protective effect on risk of breast cancer and its ER- and ER+ subtypes but increases the risk for endometrial cancer, ovarian cancer, and the endometrioid ovarian cancer subtype. This study extends the literature by addressing specifically the causal role of visceral fat on female-specific cancers.

Abstract: Background: Mounting evidence shows that adiposity increases female-specific cancer risk, but the role of body fat distribution is less clear. We used a two-sample Mendelian randomization (MR) approach to elucidate causal relations of body fat distribution to the risks of breast, endometrial and ovarian cancers and their subtypes. Methods: Body composition was assessed using segmental bioelectrical impedance analysis, yielding trunk, arm, and leg fat ratios (TFR, AFR, LFR) and BMI including 195,043 and 434,794 European women, respectively. The sample sizes for the outcomes ranged between 58,396 and 228,951 . Causal effects were estimated per one standard deviation increment in the respective exposure within the radial regression framework. Robust sensitivity analyses were performed to verify MR assumptions. In a multivariable MR setting, the proportion of risk attributable to overall and abdominal fat content was assessed. Results: TFR, which represents abdominal fat content, was associated with ovarian cancer and its clear cell and endometrioid histotypes independent of overall fat content. BMI was inversely associated with breast cancer and its ER - and ER+ subtypes, but positively with endometrial cancer and ovarian cancer, including its endometrioid histotype. These estimates were confirmed using AFR as proxy for overall body fat. Conclusions: Visceral adiposity seems to be a driver of elevated ovarian cancer risk, particularly of the endometrioid and clear cell ovarian cancer histotypes. General adiposity decreases the risk of breast cancer but increases the risk of endometrial and ovarian cancer. 
Keywords: body fat distribution; obesity; breast cancer; endometrial cancer; ovarian cancer; Mendelian randomization

\section{Introduction}

Breast cancer is the most commonly diagnosed female cancer and endometrial as well as ovarian cancer incidence rank sixth and eighth, respectively, underscoring the global impact caused by these cancers [1]. Several meta-analyses and epidemiological studies implicated obesity as a player in the development of female-specific cancers [2-4]. In observational studies associations between body mass index (BMI) as a measure of general obesity and an increased risk of postmenopausal breast [5] and a decreasing risk of premenopausal breast cancer [6] were found. Obesity is the most established risk factor for endometrial cancer $[7,8]$ and may be positively associated with ovarian cancer $[9,10]$. In recent Mendelian randomization (MR) analyses genetically predicted BMI was positively associated with endometrial cancer [11,12], but (contrary to the findings of observational studies) inversely associated with both pre- and postmenopausal breast cancer [13-15]. Another MR study reported that genetically predicted BMI was positively associated with non-high grade serous ovarian cancers but was unrelated to the more common and aggressive high-grade serous ovarian cancer [16].

A growing body of evidence suggests that body composition may play an important role in site-specific cancer development [17-19], but the data are inconclusive. Some investigations focusing on abdominal fat distribution as represented by the waist-to-hip ratio (WHR) found a positive association with breast cancer [20,21]. In a recent prospective observational study waist circumference (WC) and BMI were positively associated with endometrial and premenopausal breast cancers, but not with ovarian cancer [22]. Whether abdominal fat distribution is associated with the female-specific cancers independently of BMI remains to be clarified.

The present study is the first MR study using bioelectrical impedance analysis measurements of body fat distribution in addition to BMI as potential risk factors for female-specific cancers. We sought to generate more comprehensive and robust evidence of the impact of trunk, arm, and leg fat ratios (TFR, AFR, LFR) on breast, ovarian, as well as endometrial cancer including their histotypes based on univariable and multivariable MR analyses.

\section{Materials and Methods}

\subsection{Study Design}

A two-sample MR design allows for the assessment of a causal relationship using genome-wide association studies (GWASs) derived from different populations for the exposure and the outcome of interest. Three key assumptions need to be satisfied in a MR study: genetic instruments (i) must be associated with the respective risk factor; (ii) must not be associated with any measured or unmeasured confounder of the exposure-outcome association; and (iii) must influence the outcome only through the exposure. Details on the MR-design can be found elsewhere [23,24]. In the present study, we used both a univariable MR approach to investigate overall effects of general and abdominal fat content on femalespecific cancers and a multivariable MR (MVMR) setting to investigate the direct effects simultaneously. Analyses were based on women of European ancestry as the underlying population for exposures and outcomes.

Our analytic approach included: (i) extraction of potential genetic instruments; (ii) conducting univariable and multivariable MR analyses to assess overall and direct effects; (iii) applying several sensitivity analyses to assess different patterns of pleiotropy and validate MR assumptions. 


\subsection{Exposures Definition and Data Availability}

Regarding the amount of overall fat and for comparisons with prior MR-studies, we used BMI. To discriminate fat stored in different parts of the body, we used the fat ratio indexes TFR, AFR, LFR assessed by a segmental body impedance analysis (BIA) and calculated by dividing the fat mass in the respective body segments with total body fat mass [25]. Segmental BIA measurement is a more precise method than common anthropometric measures to discriminate between adipose and lean mass and regarding fat storage in different body compartments. Due to a strong correlation [25], AFR could be used as a measure for overall fat content and therefore replicate the results of BMI, while TFR could be utilized for investigating the impact of visceral fat content on femalespecific cancers.

Genetic instruments for BMI were obtained from a GWAS (GIANT consortium and UK Biobank) of 434,794 European women [26] (Table 1). The GWAS of fat ratio indexes TFR, AFR, and LFR was derived from 195,043 female UK Biobank participants [25].

Table 1. Overview of genome-wide association studies used in the analyses for the exposures and characteristics of the Single Nucleotide Polymorphisms (SNPs) considered as instrumental variables.

\begin{tabular}{|c|c|c|c|c|}
\hline Characteristics & BMI & AFR & TFR & LFR \\
\hline Sample size & 434,794 & 195,043 & 195,043 & 195,043 \\
\hline Consortium & GIANT, UK Biobank & UK Biobank & UK Biobank & UK Biobank \\
\hline Number of genetic instruments ${ }^{a}$ & 297 & 116 & 202 & 166 \\
\hline $\begin{array}{c}\text { Explained variance by } \\
\text { instruments }\end{array}$ & $4.63 \%$ & $3.13 \%$ & $6.77 \%$ & $5.10 \%$ \\
\hline F-statistic, mean (min; max) & $60.66(29.07 ; 941.37)$ & $51.37(29.73 ; 380.64)$ & $63.9(29.84 ; 392.04)$ & $58.51(29.73 ; 340.77)$ \\
\hline Reference & Pulit et al., 2018 [26] & $\begin{array}{l}\text { Rask-Andersen } \\
\text { et al., } 2019 \text { [25] }\end{array}$ & $\begin{array}{l}\text { Rask-Andersen et al., } \\
2019 \text { [25] }\end{array}$ & $\begin{array}{c}\text { Rask-Andersen et al., } \\
2019 \text { [25] }\end{array}$ \\
\hline
\end{tabular}

${ }^{a}$ Extraction of genetic instruments based on the threshold $p=5 \times 10^{-8}$, clumping cutoff $r^{2}=0.001$.

\subsection{Outcomes Definition and Data Availability}

As outcomes we considered breast, ovarian, and endometrial cancer. Additionally, we performed subgroup analyses of ER-positive (ER+) and ER-negative (ER-) breast cancer subtypes and the histotypes clear cell, endometrioid as well as high and low grade serous epithelial ovarian cancers.

The breast cancer GWAS was obtained from BCAC with 122,977 cases (thereof 69,501 ER+ and 21,468 ER-) and 105,974 controls [27] (Table 2). The ovarian cancer GWAS was attained from OCAC (25,509 cases and 40,941 controls) [28]. For the subgroup analyses 1366 clear cell, 2810 endometrioid, and 14,049 high or low grade serous ovarian cancer cases were available. Endometrial cancer GWAS was obtained using studies identified via the ECAC and E2C2 excluding subjects from UK Biobank with 12,270 cases and 46,126 controls [12]. There were no sample overlaps between the exposure and outcome datasets, minimizing the weak instrument bias.

Table 2. Overview of genome-wide association studies used in the analyses for the outcomes.

\begin{tabular}{|c|c|c|c|c|c|c|}
\hline Characteristics & & cer & Endometrial Cancer & \multicolumn{3}{|c|}{ Ovarian Cancer } \\
\hline Sample size & \multicolumn{2}{|c|}{228,951} & 58,396 & \multicolumn{3}{|c|}{66,450} \\
\hline Controls & \multicolumn{2}{|c|}{105,974} & 46,126 & \multicolumn{3}{|c|}{40,941} \\
\hline Cases & \multicolumn{2}{|c|}{122,977} & 12,270 & \multicolumn{3}{|c|}{25,509} \\
\hline Subtypes & ER- & $\mathrm{ER+}$ & & Clear cell & Endometrioid & $\begin{array}{l}\text { Low or high } \\
\text { grade serous }\end{array}$ \\
\hline Cases & 21,468 & 69,501 & & 1366 & 2810 & 14,049 \\
\hline Consortium & \multicolumn{2}{|c|}{$\mathrm{BCAC}$} & $\mathrm{ECAC}, \mathrm{E} 2 \mathrm{C} 2^{\mathrm{a}}$ & \multicolumn{3}{|c|}{ OCAC } \\
\hline Reference & Michaili & 2018 [27] & O'Mara et al., 2019 [12] & \multicolumn{3}{|c|}{ Phelan et al., 2017 [28] } \\
\hline
\end{tabular}




\subsection{Selection of Genetic Instruments}

We selected single nucleotide polymorphisms (SNPs) associated with the respective body composition measure below the genome-wide association threshold of $p=5 \times 10^{-8}$ and an imputation score $>0.8$. Independence of the genetic variants was ensured by performing a clumping algorithm using the stringent linkage disequilibrium (LD) cut-off $r^{2}=0.001$. For instruments missing from an outcome dataset proxy-SNPs with an LD $r^{2}>0.8$ were used.

\subsection{Statistical Power}

We calculated the statistical power according to Brion et al. [29] dependent on unknown true odds ratios (ORs) for each outcome in the range of 0.6 and 1.4 and using the type I error rate $\alpha=0.05$, outcome-specific proportions of cases and the mean explained phenotypic variance of exposures by genetic instruments $\left(R^{2}=0.05\right)$ (Figure S1).

\subsection{Statistical Analyses}

\subsubsection{Univariable MR}

As principal analysis, we performed the radial inverse-variance weighted (IVW) regression with modified second-order weights. This approach provides large statistical power and allows even for balanced pleiotropy (i.e., zero mean of random pleiotropic effects). To account for directional horizontal pleiotropy, we performed radial MR-Egger regression with modified second-order weights and tested the deviation from zero applying Egger's intercept test (Table S1).

Since the analyses were carried out iteratively, in each step we calculated SNP-specific Q-statistics and performed a leave-one-out-analysis to identify and remove outliers. Additionally, several global heterogeneity statistics were calculated and tested regarding the appropriate methods for the relationship between body composition and cancer types (Table S1).

Potential horizontal pleiotropy was assessed using robust MR approaches. The weighted median method provides a consistent estimate even if up to $50 \%$ of selected genetic instruments are invalid. The MR-PRESSO approach consists of three components, namely a global, outlier, and distortion test. The global test identifies horizontal pleiotropy based on the observed residual sum of squares (RSSobs). The outlier test corrects, if present, horizontal pleiotropy via outlier removal identified by SNP-specific RSSobs. The distortion test compares estimates before and after outlier removal. A many weak instrument analysis was done using the Robust Adjusted Profile Score, allowing instruments to be weak.

\subsubsection{Multivariable MR}

To compare the direct effects and separate out what proportion of female-specific cancer risk is due to general and visceral fat content, we performed MVMR analyses. AFR and TFR were utilized as proxies for the degree of overall and abdominal fat content, respectively. The conditional F-statistics were at least 25.9 implicating absence of weak instrument bias. Horizontal pleiotropy was quantified and tested using the modified Cochran's Q-statistics, while directional pleiotropy was investigated using MR-Egger intercept tests (Table S2). Causal effect estimates were obtained using the robust IVW approach with multiplicative random effects as the main method and Median and MREgger methods as sensitivity analyses. Effect estimation after outlier assessment and deletion was done by the MR-Lasso procedure. Finally, we used the Q-minimization approach to calculate robust point estimates that account for both weak instruments and substantial heterogeneity.

All estimates depict ORs per one standard deviation (SD) increments in the exposures. The type I error was set to $\alpha=0.01$ when investigating outliers based on Q-statistics within the radial framework and to $\alpha=0.05$ otherwise. To correct for multiple testing the Benjamini-Hochberg procedure was applied. Analyses were performed using mainly the packages TwoSampleMR (0.5.5), MendelianRandomization (0.4.3), RadialMR (1.0), and 
MRPRESSO (1.0) of the statistical Software R (Version: 4.0.3) (the R Foundation is seated in Vienna, Austria).

\section{Results}

\subsection{Instrument Strength and Statistical Power}

As genetic instruments we selected 297 BMI-, 202 TFR-, 116 AFR-, and 166 LFRrelated SNPs (Table 1). For BMI $4.6 \%$ of the phenotypic variance was explained by the genetic instruments. For TFR, AFR, and LFR the explained variances were $6.8 \%, 3.1 \%$, and $5.1 \%$, respectively. The SNP-specific F-statistics ranging from 29.1 (BMI) to 941.4 (BMI) (Tables S3-S34), were clearly above the traditional threshold of 10, indicating absence of weak instrument bias. We determined a statistical power $\geq 0.8$ when the true ORs for the impact of body fat indexes on breast, ovarian, and endometrial cancers were expected to be $\leq 0.95$ or $\geq 1.05, \leq 0.90$ or $\geq 1.10, \leq 0.88$ or $\geq 1.12$, respectively. The statistical power in the subgroup analyses were slightly lower for ER+ and ER- breast cancers and (high or low grade) serous ovarian cancer.

\subsection{Effects of Body Fat on Breast Cancer and Its Subtypes}

The Radial IVW models with modified second-order weights as the main method (point estimates are subsequently referred to as OR_IVW and are given per $1 \mathrm{SD}$ increment of the respective exposure) found an inverse association between BMI and breast cancer $\left(\mathrm{OR}_{\mathrm{IVW}}=0.86 ; 95 \% \mathrm{CI}:[0.81,0.91]\right)$ (Figure 1$)$. These results were verified using the AFR as proxy for general fat content $\left(\mathrm{OR}_{\mathrm{IVW}}=0.90 ; 95 \% \mathrm{CI}\right.$ : [0.84, 0.96]). However, TFR as a proxy for visceral fat was positively but weakly associated with breast $\left(\mathrm{OR}_{\mathrm{IVW}}=1.05 ; 95 \% \mathrm{CI}\right.$ : $[1.00,1.10])$. All corresponding scatterplots of the SNP-effects on both the exposures and outcomes can be found at Figures S2-S5.

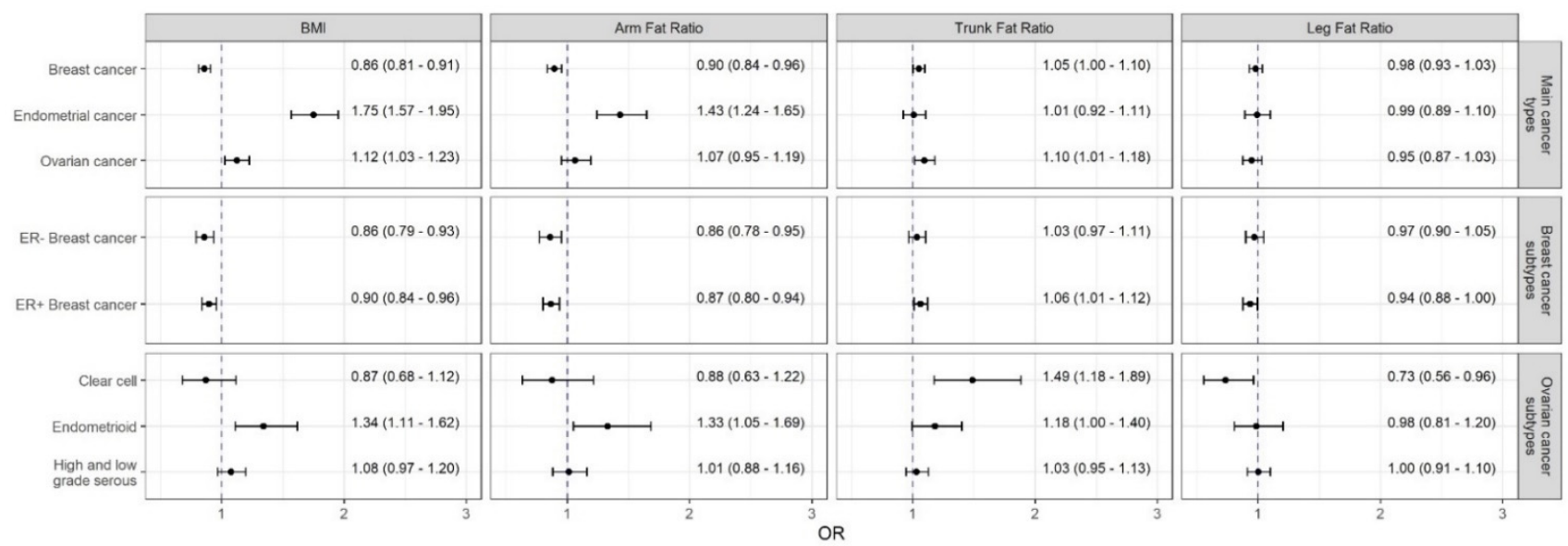

Figure 1. Causal estimates (odds ratios and 95\% confidence intervals per one standard deviation change in the respective exposure) of the impact of obesity measures on female-specific cancers obtained by the radial inverse-variance weighted (IVW) approach with modified second-order weights. Abbreviations: FE, fixed effects; IVW (Mod. 2nd), inverse-variance weighted model with modified second-order weights; RAPS, Robust Adjusted Profile Score; RE, random effects; PRESSO, Pleiotropy RESidual Sum and Outlier.

Although there was evidence for substantial heterogeneity (suggesting the Radial MREgger with multiplicative random effects as the appropriate method) (Table S1), the strong results (i.e., impact of overall fat content on breast cancer) were supported by sensitivity analyses using robust methods (Figure S6).

After mutual adjustment, the inverse effect of AFR with breast cancer (Robust IVW $\mathrm{OR}=0.86 ; 95 \% \mathrm{CI}:[0.77,0.96])$ did not changed remarkably, while the positive association between TFR and breast cancer attenuated in the multivariable setting (Figure 2). 
A
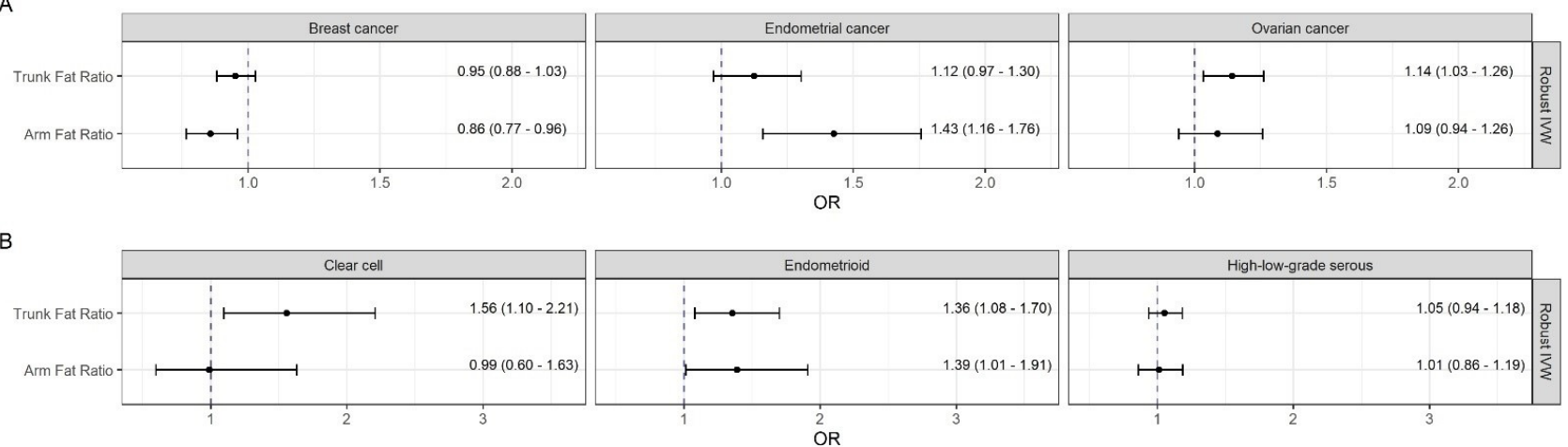

C

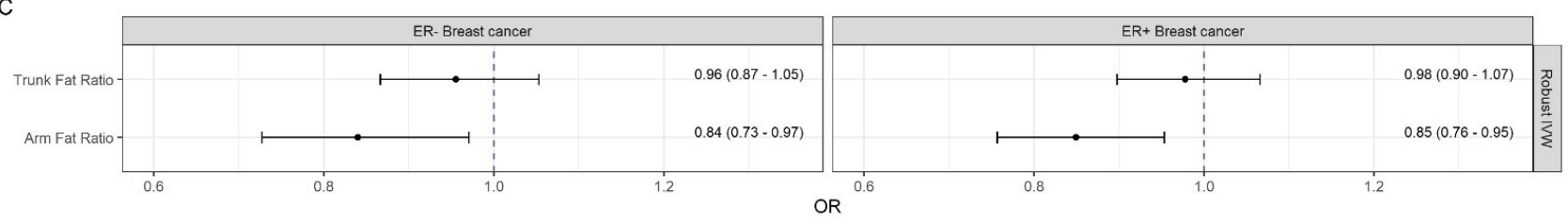

Figure 2. Mutually adjusted causal estimates (odds ratios and 95\% confidence intervals per one SD change in the appropriate exposure) from multivariable Mendelian randomization analyses of genetically predicted trunk and arm fat ratios on female-specific cancers. Abbreviations: IVW, inverse-variance weighted; OR, odds ratio.

Subgroup analyses revealed consistent inverse associations of genetically predicted BMI and AFR with ER- $\left(\mathrm{OR}_{\mathrm{IVW}}=0.86 ; 95 \% \mathrm{CI}\right.$ : $[0.79,0.93]$ and $\mathrm{OR}_{\mathrm{IVW}}=0.86 ; 95 \% \mathrm{CI}$ : $[0.78,0.95])$ as well as ER+ breast cancer $\left(\mathrm{OR}_{\mathrm{IVW}}=0.90 ; 95 \% \mathrm{CI}\right.$ : $[0.84,0.96]$ and $\mathrm{OR}_{\mathrm{IVW}}=0.87$; 95\% CI: [0.80, 0.94]) (Figure 1) despite heterogeneity in the relationships with the latter. TFR was positively and LFR inversely related to ER+ (with inconsistent Egger estimates) but not to ER - breast cancer (Figure S7).

The multivariable analyses confirmed the protective effects of AFR on ER- (Robust IVW OR $=0.84 ; 95 \%$ CI: $[0.73,0.97]$ ) and ER+ breast cancer (Robust IVW OR $=0.85 ; 95 \%$ CI: $[0.76,0.95])$, while the formerly association between TFR and ER+ breast cancer could not be substantiated.

\subsection{Effects of Body Fat on Endometrial Cancer}

Both genetically predicted BMI and AFR were robustly associated with endometrial cancer with $\mathrm{OR}_{\mathrm{IVW}}=1.75$ (95\% CI: $\left.[1.57,1.95]\right)$ and $\mathrm{OR}_{\mathrm{IVW}}=1.43(95 \% \mathrm{CI}:[1.24,1.65])$, respectively (Figure 1). These effects were supported by the multivariable analysis, where the effect of AFR on endometrial cancer were adjusted for TFR (Robust IVW OR $=1.43$; 95\% CI: $[1.16,1.76]$ ) (Figure 2). No further notable associations were observed.

\subsection{Effects of Body Fat on Ovarian Cancer and Its Histotypes}

Although there was not a clear association with ovarian cancer, consistently positive relations were observed of both BMI and AFR to endometrioid ovarian cancer, with $\mathrm{OR}_{\mathrm{IVW}}=1.34(95 \% \mathrm{CI}:[1.11,1.62])$ and $\mathrm{OR}_{\mathrm{IVW}}=1.33$ (95\% CI: $\left.[1.05,1.69]\right)$, respectively (Figure 1). Regarding the visceral fat content, genetically predicted TFR was positively associated with ovarian cancer $\left(\mathrm{OR}_{\mathrm{IVW}}=1.10 ; 95 \% \mathrm{CI}\right.$ : $\left.[1.01,1.18]\right)$ as well as the clear cell $\left(\mathrm{OR}_{\mathrm{IVW}}=1.49 ; 95 \% \mathrm{CI}:[1.18,1.89]\right)$, and endometrioid histotypes $\left(\mathrm{OR}_{\mathrm{IVW}}=1.18 ; 95 \% \mathrm{CI}\right.$ : $[1.00,1.40]$. Apart from an inverse association between LFR and clear cell ovarian cancer $\left(\mathrm{OR}_{\mathrm{IVW}}=0.73 ; 95 \% \mathrm{CI}:[0.56,0.96]\right)$, there were no further notable relations. Again, the sensitivity analyses supported these findings (Figure S8).

In the multivariable setting, the association between AFR and the endometrioid ovarian cancer remained positive (Robust IVW OR $=1.39 ; 95 \%$ CI: [1.01, 1.91]). The effect of TFR on ovarian cancer and its clear cell and endometrioid histotypes were partly even stronger with Robust IVW OR $=1.14$ (95\% CI: $[1.03,1.26])$, OR $=1.56$ (95\% CI: [1.10, 2.21]), and $\mathrm{OR}=1.36(95 \% \mathrm{CI}:[1.08,1.70])$, respectively. 
Although no directional pleiotropy was detected by MR-Egger intercept tests, there was evidence for substantial heterogeneity quantified by Q-statistics in the multivariable models (Table S2). However, all results were supported by sensitivity analyses (Figures S9-S11). Especially the heterogeneity robust Q-minimization models produced consistent point estimates to the robust IVW approach as the main method.

\section{Discussion}

Our MR study showed that visceral adiposity increased the risk of breast cancer, mainly ER+ breast cancer, and the risk of ovarian cancer, mainly clear cell ovarian cancer. Relations of genetically predicted visceral adiposity to breast and endometrial cancers were weaker than those with general adiposity. However, visceral adiposity appears to play a more important role than general adiposity for the risk of ovarian cancer, in particular the clear cell and endometrioid cancer subtypes. The findings confirmed a causal protective effect of general adiposity on breast cancer risk. This effect could be ascribed to associations with both ER- and ER+ breast cancers and was confirmed in multivariable analyses. In contrast, general adiposity was a strong causal risk factor for endometrial cancer and a weaker causal risk factor for ovarian cancer, in particular for the endometrioid histotype. LFR was not related to overall cancers but showed effects in the same direction as AFR on ER+ breast cancer and clear cell ovarian cancer.

Epidemiologic studies reported a positive relationship between obesity and postmenopausal breast cancer and an inverse association with premenopausal breast cancer [30-33]. One study using data from two chemoprevention trials found a positive association in premenopausal women [34]. In contrast, two prior MR studies showed that adult BMI is inversely related to postmenopausal breast cancer $[13,14]$. We also found an inverse causal effect of overall fat content on breast cancer risk; however, we were unable to distinguish between pre- and postmenopausal breast cancers. The apparent adverse effect of adult body size on breast cancer risk may be attributable to a large body size in childhood that persists into adulthood [35]. Of note, childhood BMI showed inverse associations with both premenopausal and postmenopausal breast cancer $[36,37]$. Because adult weight gain is a strong predictor of postmenopausal breast cancer risk [38], the positive association between adult BMI and postmenopausal breast cancer shown in epidemiologic studies is likely due to the effect of weight gain in adulthood. Weight gain may better reflect the dynamics of changes in body shape over time, with increased visceral and metabolically active fat accumulation, especially during menopause [39]. According to the WCRF, abdominal obesity and weight gain in adulthood convincingly increases the risk of postmenopausal breast cancer [31]. Our finding that genetically predicted TFR showed a positive effect on ER+ breast cancer suggests that body fat distribution plays a particular role in postmenopausal breast cancer. Supporting the assessment of the IARC Working Group, [30], we showed that an increasing BMI and AFR had a strongly positive effect on endometrial cancer. However, we found no evidence for a causal link between abdominal adiposity and endometrial cancer; this is in contrast to umbrella reviews $[3,4,31]$, in which the association between WC and WHR and total endometrial cancer was supported by strong evidence. Our results support the findings of recent MR studies, where genetically elevated BMI, but not WHR, was found to be causally related to endometrial cancer risk $[11,40]$.

The evidence relating body fatness to ovarian cancer and its subtypes is inconclusive [41]. The WCRF CUP graded the relationship as probably causal [31] and the IARC suggested there is strong evidence for a positive association between obesity and ovarian cancer [30]. Likewise, a systematic review and meta-analysis reported an increased risk of ovarian cancer with higher BMI, and a marginally significant positive association with WC, but no association with hip circumference or WHR [42]. An umbrella review of doseresponse meta-analyses rated the evidence for an association between BMI and ovarian cancer as weak [43], but another umbrella review [2] rated the evidence as suggestive only [2]. Using a MR approach, Gao et al. [14] found a positive association between BMI 
and ovarian cancer, which is in line with our results; contrary, a one-sample MR study found no association between both BMI and fat mass and ovarian cancer [44]. However, our study was two-sampled, included more cases, and used larger GWAS with 201 more genetic instruments for the analyses resulting in higher statistical power. We further showed a positive association between TFR and ovarian cancer, in particular for the endometrioid and clear cell histotypes, a result that extends the existing evidence and may contribute to better understanding of the pathogenesis of this cancer.

Obese adipose tissue seems to create a pro-oncogenic environment. Specific biological mechanisms to explore the link between obesity and cancer risk are centered along metabolic and endocrine consequences of body fat accumulation. Changes in the metabolism of sex hormones, insulin and insulin-like growth factor signaling, inflammatory effects and Adipokine-related effects were identified. According to an IARC working group, the evidence for a role of sex hormones and chronic inflammation was judged as strong, while the evidence for insulin and IGF was moderate [30]. Among other factors, obesity-related changes in the tumor microenvironment, cellular perturbations, and the intestinal microbiome are likely to affect cancer development [45]. In addition, obesityrelated changes in anti-tumor immunity and angiogenesis were observed [46]. Biological mechanisms that could explain the inverse effects of BMI and AFR on the development of breast cancer and its subtypes are widely unknown.

Our study has a number of considerable strengths. Using both BMI and AFR we could replicate the findings of a previous MR study, which provided support for our estimates. While BMI approximates total fat content, the fat ratio indexes used in our study discriminated between adipose and lean mass and are therefore more reflective of actual fat mass and body fat distribution. All exposures were modelled as continuous variables to avoid residual confounding and to boost statistical power. Sufficiently large F-statistics reduced the potential for weak instrument bias. The broad range of robust methods allowed us to strengthen our findings by considering different patterns of pleiotropy. The multivariable approach allowed us to distinguish between the direct effects of overall and abdominal fat content.

Our study also has certain limitations. TFR considers the fat content of the entire torso rather than that of the abdomen only, which may have underestimated causal associations. MR analyses assume relationships to be linear. Quantitative estimates may be misleading if the true relationship is non-linear; although estimates are still reflective of the presence and direction of the population-averaged causal effect [47]. Our analyses were based on European women and thus, results cannot be transferred to other ethnicities. Finally, we were unable to distinguish between pre- and postmenopausal breast cancer.

\section{Conclusions}

In conclusion, our MR study shows a protective effect of general adiposity on risk of breast cancer and its ER- and ER+ subtypes. In contrast, general adiposity increases the risk for endometrial cancer, ovarian cancer, and the endometrioid ovarian cancer subtype. Visceral adiposity is related to an increased risk for ovarian cancer and its endometrioid and clear cell subtypes and is suggestively associated with increased risk of breast cancer and its ER+ subtype. These results extend the literature by addressing the role of visceral adiposity regarding female-specific cancers and support previous findings on general adiposity. Thus, it can be concluded that different body fat distributions lead to different risk profiles and not obesity per se, but rather body fat distribution should be taken into consideration in connection with the risk of female-specific cancers.

Supplementary Materials: The following are available online at https:/ / www.mdpi.com/article/10 .3390/ cancers13205053/s1, Figure S1: Power analysis, Figure S2: Sensitivity analyses of univariable Mendelian randomization for main cancer types, Figure S3: Sensitivity analyses of univariable Mendelian randomization for breast cancer subtypes, Figure S4: Sensitivity analyses of univariable Mendelian randomization for ovarian cancer subtypes, Figure S5: Sensitivity analyses of multivariable Mendelian randomization for main cancer types, Figure S6: Sensitivity analyses of multivariable 
Mendelian randomization for breast cancer subtypes, Figure S7: Sensitivity analyses of multivariable Mendelian randomization for ovarian cancer subtypes, Figure S8: Scatter plot matrix of SNP effects on both body mass index and female-specific cancers, Figure S9: Scatter plot matrix of SNP effects on both arm fat ratio and female-specific cancers, Figure S10: Scatter plot matrix of SNP effects on both trunk fat ratio and female-specific cancers, Figure S11: Scatter plot matrix of SNP effects on both leg fat ratio and female-specific cancers, Table S1: Heterogeneity statistics from univariable Mendelian randomization analyses, Table S2: Instrument strength and heterogeneity from multivariable Mendelian randomization, Table S3: BMI related SNP-associations with breast cancer, Table S4: BMI related SNP-associations with endometrial cancer, Table S5: BMI related SNP-associations with ovarian cancer, Table S6: BMI related SNP-associations with ER- breast cancer, Table S7: BMI related SNP-associations with ER+ breast cancer, Table S8: BMI related SNP-associations with clear cell ovarian cancer, Table S9: BMI related SNP-associations with endometrioid ovarian cancer, Table S10: BMI related SNP-associations with high or low serous ovarian cancer, Table S11: Arm fat ratio related SNP-associations with breast cancer, Table S12: Arm fat ratio related SNP-associations with endometrial cancer, Table S13: Arm fat ratio related SNP-associations with ovarian cancer, Table S14: Arm fat ratio related SNP-associations with ER- breast cancer, Table S15: Arm fat ratio related SNPassociations with ER+ breast cancer, Table S16: Arm fat ratio related SNP-associations with clear cell ovarian cancer, Table S17: Arm fat ratio related SNP-associations with endometrioid ovarian cancer, Table S18: Arm fat ratio related SNP-associations with high or low serous ovarian cancer, Table S19: Trunk fat ratio related SNP-associations with breast cancer, Table S20: Trunk fat ratio related SNP-associations with endometrial cancer, Table S21: Trunk fat ratio related SNP-associations with ovarian cancer, Table S22: Trunk fat ratio related SNP-associations with ER- breast cancer, Table S23: Trunk fat ratio related SNP-associations with ER+ breast cancer, Table S24: Trunk fat ratio related SNP-associations with clear cell ovarian cancer, Table S25: Trunk fat ratio related SNPassociations with endometrioid ovarian cancer, Table S26: Trunk fat ratio related SNP-associations with high or low serous ovarian cancer, Table S27: Leg fat ratio related SNP-associations with breast cancer, Table S28: Leg fat ratio related SNP-associations with endometrial cancer, Table S29: Leg fat ratio related SNP-associations with ovarian cancer, Table S30: Leg fat ratio related SNP-associations with ER- breast cancer, Table S31: Leg fat ratio related SNP-associations with ER+ breast cancer, Table S32: Leg fat ratio related SNP-associations with clear cell ovarian cancer, Table S33: Leg fat ratio related SNP-associations with endometrioid ovarian cancer, Table S34: Leg fat ratio related SNP-associations with high or low serous ovarian cancer.

Author Contributions: D.F. analyzed the data and created the tables and plots. C.M. did the literature research and contributed together with D.F. and H.B. to the data collection. T.A.O. conducted and provided the GWAS for endometrial cancer. D.F., C.M., J.L., T.A.O., M.L., H.B. and S.-E.B. wrote the manuscript and contributed to data interpretation. J.L., C.M., M.L. and S.-E.B. reviewed the final manuscript. J.L. and C.M. supervised the project. All authors have read and agreed to the published version of the manuscript.

Funding: The authors did not receive funding for this study. Funding information of the genomewide association studies is specified in the cited studies.

Institutional Review Board Statement: Not applicable, since the study is based on summary-level data. In all original studies, ethical approval had been obtained.

Informed Consent Statement: Not applicable, since the study is based on summary-level data. In all original studies, informed consent was obtained from all subjects involved.

Data Availability Statement: The present study is based on summary-level data that have been made publically available. Summary data from genome-wide association studies for the BMI (Pulit et al.) is available at https: / / zenodo.org/record/1251813\#.XxgQ2J5KiUl (accessed on 26 March 2021); Data for the ratios TFR, AFR, and LFR (Rask-Andersen et al.) can be obtained from https: / myfiles.uu.se/ ssf/s/readFile/share/3993/1270878243748486898/publicLink/GWAS_summary_stats_ratios.zip (accessed on 12 March 2021); Summary level data for breast cancer and its subgroups can be obtained from the BCAC Consortium (http:/ / bcac.ccge.medschl.cam.ac.uk/bcacdata/oncoarray/oncoarrayand-combined-summary-result/gwas-summary-results-breast-cancer-risk-2017) (accessed on 3 February 2021) and for ovarian cancer and its subgroups from MRC IEU OpenGWAS Project database (https:/ /gwas.mrcieu.ac.uk) (accessed on 10 February 2021). Endometrial cancer summary data (O'Mara et al.) restricted to subjects apart from UK Biobank is available on request from authors. 
Acknowledgments: We thank all institutions for providing the publically available genome-wide association studies used in this study.

Conflicts of Interest: The authors declare no conflict of interest.

\section{References}

1. Sung, H.; Ferlay, J.; Siegel, R.L.; Laversanne, M.; Soerjomataram, I.; Jemal, A.; Bray, F. Global Cancer Statistics 2020: Globocan Estimates of Incidence and Mortality Worldwide for 36 Cancers in 185 Countries. CA Cancer J. Clin. 2021, 71, 209-249. [CrossRef]

2. Kalliala, I.; Markozannes, G.; Gunter, M.J.; Paraskevaidis, E.; Gabra, H.; Mitra, A.; Terzidou, V.; Bennett, P.; Martin-Hirsch, P.; Tsilidis, K.K.; et al. Obesity and Gynaecological and Obstetric Conditions: Umbrella Review of the Literature. BMJ 2017,359, j4511. [CrossRef] [PubMed]

3. Kyrgiou, M.; Kalliala, I.; Markozannes, G.; Gunter, M.J.; Paraskevaidis, E.; Gabra, H.; Martin-Hirsch, P.; Tsilidis, K.K. Adiposity and Cancer at Major Anatomical Sites: Umbrella Review of the Literature. BMJ 2017, 356, j477. [CrossRef] [PubMed]

4. Raglan, O.; Kalliala, I.; Markozannes, G.; Cividini, S.; Gunter, M.J.; Nautiyal, J.; Gabra, H.; Paraskevaidis, E.; Martin-Hirsch, P.; Tsilidis, K.K.; et al. Risk Factors for Endometrial Cancer: An Umbrella Review of the Literature. Int. J. Cancer 2019, 145, 1719-1730. [CrossRef] [PubMed]

5. Carpenter, C.L.; Ross, R.K.; Paganini-Hill, A.; Bernstein, L. Effect of Family History, Obesity and Exercise on Breast Cancer Risk among Postmenopausal Women. Int. J. Cancer 2003, 106, 96-102. [CrossRef] [PubMed]

6. Bhaskaran, K.; Douglas, I.; Forbes, H.; dos-Santos-Silva, I.; Leon, D.A.; Smeeth, L. Body-Mass Index and Risk of 22 Specific Cancers: A Population-Based Cohort Study of 5.24 Million Uk Adults. Lancet 2014, 384, 755-765. [CrossRef]

7. Lu, L.; Risch, H.; Irwin, M.L.; Mayne, S.T.; Cartmel, B.; Schwartz, P.; Rutherford, T.; Yu, H. Long-Term Overweight and Weight Gain in Early Adulthood in Association with Risk of Endometrial Cancer. Int. J. Cancer 2011, 129, 1237-1243. [CrossRef]

8. Onstad, M.A.; Schmandt, R.E.; Lu, K.H. Addressing the Role of Obesity in Endometrial Cancer Risk, Prevention, and Treatment. J. Clin. Oncol. 2016, 34, 4225-4230. [CrossRef]

9. Aune, D.; Rosenblatt, D.A.N.; Chan, D.S.; Vingeliene, S.; Abar, L.; Vieira, A.R.; Greenwood, D.C.; Bandera, E.V.; Norat, T. Anthropometric Factors and Endometrial Cancer Risk: A Systematic Review and Dose-Response Meta-Analysis of Prospective Studies. Ann. Oncol. 2015, 26, 1635-1648. [CrossRef]

10. Wentzensen, N.; Poole, E.M.; Trabert, B.; White, E.; Arslan, A.A.; Patel, A.V.; Setiawan, V.W.; Visvanathan, K.; Weiderpass, E.; Adami, H.O.; et al. Ovarian Cancer Risk Factors by Histologic Subtype: An Analysis from the Ovarian Cancer Cohort Consortium. J. Clin. Oncol. 2016, 34, 2888-2898. [CrossRef]

11. Painter, J.N.; O’Mara, T.A.; Marquart, L.; Webb, P.M.; Attia, J.; Medland, S.E.; Cheng, T.; Dennis, J.; Holliday, E.G.; McEvoy, M.; et al. Genetic Risk Score Mendelian Randomization Shows That Obesity Measured as Body Mass Index, but Not Waist:Hip Ratio, Is Causal for Endometrial Cancer. Cancer Epidemiol. Biomark. Prev. 2016, 25, 1503-1510. [CrossRef] [PubMed]

12. O'Mara, T.A.; Glubb, D.M.; Amant, F.; Annibali, D.; Ashton, K.; Attia, J.; Auer, P.L.; Beckmann, M.W.; Black, A.; Bolla, M.K.; et al. Identification of Nine New Susceptibility Loci for Endometrial Cancer. Nat. Commun. 2018, 9, 3166. [CrossRef]

13. Guo, Y.; Andersen, S.W.; Shu, X.O.; Michailidou, K.; Bolla, M.K.; Wang, Q.; Garcia-Closas, M.; Milne, R.L.; Schmidt, M.K.; Chang-Claude, J. Borresen-Dale Genetically Predicted Body Mass Index and Breast Cancer Risk: Mendelian Randomization Analyses of Data from 145,000 Women of European Descent. PLoS Med. 2016, 13, e1002105. [CrossRef] [PubMed]

14. Gao, C.; Patel, C.J.; Michailidou, K.; Peters, U.; Gong, J.; Schildkraut, J.; Schumacher, F.R.; Zheng, W.; Boffetta, P.; Stucker, I.; et al. Mendelian Randomization Study of Adiposity-Related Traits and Risk of Breast, Ovarian, Prostate, Lung and Colorectal Cancer. Int. J. Epidemiol. 2016, 45, 896-908. [CrossRef] [PubMed]

15. Shu, X.; Wu, L.; Khankari, N.K.; Shu, X.O.; Wang, T.J.; Michailidou, K.; Bolla, M.K.; Wang, Q.; Dennis, J.; Milne, R.L.; et al. Associations of Obesity and Circulating Insulin and Glucose with Breast Cancer Risk: A Mendelian Randomization Analysis. Int. J. Epidemiol. 2019, 48, 795-806. [CrossRef]

16. Dixon, S.C.; Nagle, C.M.; Thrift, A.P.; Pharoah, P.D.; Pearce, C.L.; Zheng, W.; Painter, J.N.; Group, A.; Cancer, S.A.; Chenevix-Trench, G.; et al. Adult Body Mass Index and Risk of Ovarian Cancer by Subtype: A Mendelian Randomization Study. Int. J. Epidemiol. 2016, 45, 884-895. [CrossRef]

17. Harding, J.L.; Shaw, J.E.; Anstey, K.J.; Adams, R.; Balkau, B.; Brennan-Olsen, S.L.; Briffa, T.; Davis, T.M.; Davis, W.A.; Dobson, A.; et al. Comparison of Anthropometric Measures as Predictors of Cancer Incidence: A Pooled Collaborative Analysis of 11 Australian Cohorts. Int. J. Cancer 2015, 137, 1699-1708. [CrossRef]

18. Freisling, H.; Arnold, M.; Soerjomataram, I.; O’Doherty, M.G.; Ordonez-Mena, J.M.; Bamia, C.; Kampman, E.; Leitzmann, M.; Romieu, I.; Kee, F.; et al. Comparison of General Obesity and Measures of Body Fat Distribution in Older Adults in Relation to Cancer Risk: Meta-Analysis of Individual Participant Data of Seven Prospective Cohorts in Europe. Br. J. Cancer 2017, 116, 1486-1497. [CrossRef]

19. Arnold, M.; Leitzmann, M.; Freisling, H.; Bray, F.; Romieu, I.; Renehan, A.; Soerjomataram, I. Obesity and Cancer: An Update of the Global Impact. Cancer Epidemiol. 2016, 41, 8-15. [CrossRef]

20. Huang, Z.; Willett, W.C.; Colditz, G.A.; Hunter, D.J.; Manson, J.E.; Rosner, B.; Speizer, F.E.; Hankinson, S.E. Waist Circumference, Waist:Hip Ratio, and Risk of Breast Cancer in the Nurses' Health Study. Am. J. Epidemiol. 1999, 150, 1316-1324. [CrossRef]

21. White, A.J.; Nichols, H.B.; Bradshaw, P.T.; Sandler, D.P. Overall and Central Adiposity and Breast Cancer Risk in the Sister Study. Cancer 2015, 121, 3700-3708. [CrossRef] 
22. Recalde, M.; Davila-Batista, V.; Diaz, Y.; Leitzmann, M.; Romieu, I.; Freisling, H.; Duarte-Salles, T. Body Mass Index and Waist Circumference in Relation to the Risk of 26 Types of Cancer: A Prospective Cohort Study of 3.5 Million Adults in Spain. BMC Med. 2021, 19, 10. [CrossRef]

23. Davies, N.M.; Holmes, M.V.; Smith, G.D. Reading Mendelian Randomisation Studies: A Guide, Glossary, and Checklist for Clinicians. BMJ 2018, 362. [CrossRef]

24. Burgess, S.; Thompson, S.G. Multivariable Mendelian Randomization: The Use of Pleiotropic Genetic Variants to Estimate Causal Effects. Am. J. Epidemiol. 2015, 181, 251-260. [CrossRef] [PubMed]

25. Rask-Andersen, M.; Karlsson, T.; Ek, W.E.; Johansson, A. Genome-Wide Association Study of Body Fat Distribution Identifies Adiposity Loci and Sex-Specific Genetic Effects. Nat. Commun. 2019, 10, 339. [CrossRef]

26. Pulit, S.L.; Stoneman, C.; Morris, A.P.; Wood, A.R.; Glastonbury, C.A.; Tyrrell, J.; Yengo, L.; Ferreira, T.; Marouli, E.; Ji, Y.; et al. Meta-Analysis of Genome-Wide Association Studies for Body Fat Distribution in 694649 Individuals of European Ancestry. Hum. Mol. Genet. 2019, 28, 166-174. [CrossRef] [PubMed]

27. Michailidou, K.; Lindstrom, S.; Dennis, J.; Beesley, J.; Hui, S.; Kar, S.; Lemacon, A.; Soucy, P.; Glubb, D.; Rostamianfar, A.; et al. Association Analysis Identifies 65 New Breast Cancer Risk Loci. Nature 2017, 551, 92-94. [CrossRef]

28. Phelan, C.M.; Kuchenbaecker, K.B.; Tyrer, J.P.; Kar, S.P.; Lawrenson, K.; Winham, S.J.; Dennis, J.; Pirie, A.; Riggan, M.J.; Chornokur, G.; et al. Identification of 12 New Susceptibility Loci for Different Histotypes of Epithelial Ovarian Cancer. Nat. Genet. 2017, 49, 680-691. [CrossRef] [PubMed]

29. Brion, M.-J.A.; Shakhbazov, K.; Visscher, P.M. Calculating Statistical Power in Mendelian Randomization Studies. Int. J. Epidemiol. 2012, 42, 1497-1501. [CrossRef]

30. Lauby-Secretan, B.; Scoccianti, C.; Loomis, D.; Grosse, Y.; Bianchini, F.; Straif, K.; Group International Agency for Research on Cancer Handbook Working. Body Fatness and Cancer-Viewpoint of the Iarc Working Group. N. Engl. J. Med. 2016, 375, 794-798. [CrossRef]

31. World Cancer Research Fund/American Institute for Cancer Research. Continuous Update Project Expert Report 2018. Body Fatness and Weight Gain and the Risk of Cancer. Available online: https:/ / www.dietandcancerreport.org (accessed on 7 May 2021).

32. Premenopausal Breast Cancer Collaborative Group; Schoemaker, M.J.; Nichols, H.B.; Wright, L.B.; Brook, M.N.; Jones, M.E.; O'Brien, K.M.; Adami, H.O.; Baglietto, L.; Bernstein, L.; et al. Association of Body Mass Index and Age with Subsequent Breast Cancer Risk in Premenopausal Women. JAMA Oncol. 2018, 4, e181771.

33. Neuhouser, M.L.; Aragaki, A.K.; Prentice, R.L.; Manson, J.E.; Chlebowski, R.; Carty, C.L.; Ochs-Balcom, H.M.; Thomson, C.A.; Caan, B.J.; Tinker, L.F.; et al. Overweight, Obesity, and Postmenopausal Invasive Breast Cancer Risk: A Secondary Analysis of the Women's Health Initiative Randomized Clinical Trials. JAMA Oncol. 2015, 1, 611-621. [CrossRef] [PubMed]

34. Cecchini, R.S.; Costantino, J.P.; Cauley, J.A.; Cronin, W.M.; Wickerham, D.L.; Land, S.R.; Weissfeld, J.L.; Wolmark, N. Body Mass Index and the Risk for Developing Invasive Breast Cancer among High-Risk Women in Nsabp P-1 and Star Breast Cancer Prevention Trials. Cancer Prev. Res. 2012, 5, 583-592. [CrossRef] [PubMed]

35. Richardson, T.G.; Sanderson, E.; Elsworth, B.; Tilling, K.; Smith, G.D. Use of Genetic Variation to Separate the Effects of Early and Later Life Adiposity on Disease Risk: Mendelian Randomisation Study. BMJ 2020, 369, m1203. [CrossRef] [PubMed]

36. Baer, H.J.; Tworoger, S.S.; Hankinson, S.E.; Willett, W.C. Body Fatness at Young Ages and Risk of Breast Cancer throughout Life. Am. J. Epidemiol. 2010, 171, 1183-1194. [CrossRef]

37. Baer, H.J.; Colditz, G.A.; Rosner, B.; Michels, K.B.; Rich-Edwards, J.W.; Hunter, D.J.; Willett, W.C. Body Fatness During Childhood and Adolescence and Incidence of Breast Cancer in Premenopausal Women: A Prospective Cohort Study. Breast Cancer Res. 2005, 7, R314. [CrossRef]

38. Sandholt, C.H.; Allin, K.H.; Toft, U.; Borglykke, A.; Ribel-Madsen, R.; Sparso, T.; Justesen, J.M.; Harder, M.N.; Jorgensen, T.; Hansen, T.; et al. The Effect of Gwas Identified Bmi Loci on Changes in Body Weight among Middle-Aged Danes during a Five-Year Period. Obesity 2014, 22, 901-908. [CrossRef]

39. Keum, N.; Greenwood, D.C.; Lee, D.H.; Kim, R.; Aune, D.; Ju, W.; Hu, F.B.; Giovannucci, E.L. Adult Weight Gain and AdiposityRelated Cancers: A Dose-Response Meta-Analysis of Prospective Observational Studies. J. Natl. Cancer Inst. 2015, 107. [CrossRef]

40. Nead, K.T.; Sharp, S.J.; Thompson, D.J.; Painter, J.N.; Savage, D.B.; Semple, R.K.; Barker, A.; Study, G.A.N.E.C.; Perry, J.R.; Attia, J.; et al. Evidence of a Causal Association between Insulinemia and Endometrial Cancer: A Mendelian Randomization Analysis. J. Natl. Cancer Inst. 2015, 107. [CrossRef]

41. World Cancer Research Fund/American Institute for Cancer Research. Food, Nutrition, Physical Activity and the Prevention of Cancer: A Global Perspective. Available online: https:/ / www.wcrf.org/dietandcancer (accessed on 7 May 2021).

42. Aune, D.; Rosenblatt, D.A.N.; Chan, D.S.; Abar, L.; Vingeliene, S.; Vieira, A.R.; Greenwood, D.C.; Norat, T. Anthropometric Factors and Ovarian Cancer Risk: A Systematic Review and Nonlinear Dose-Response Meta-Analysis of Prospective Studies. Int. J. Cancer 2015, 136, 1888-1898. [CrossRef]

43. Choi, E.K.; Park, H.B.; Lee, K.H.; Park, J.H.; Eisenhut, M.; van der Vliet, H.J.; Kim, G.; Shin, J.I. Body Mass Index and 20 Specific Cancers: Re-Analyses of Dose-Response Meta-Analyses of Observational Studies. Ann. Oncol. 2018, 29, 749-757. [CrossRef] [PubMed]

44. Vithayathil, M.; Carter, P.; Kar, S.; Mason, A.M.; Burgess, S.; Larsson, S.C. Body Size and Composition and Site-Specific Cancers in Uk Biobank: A Mendelian Randomisation Study. bioRxiv 2020. [CrossRef] 
45. Avgerinos, K.I.; Spyrou, N.; Mantzoros, C.S.; Dalamaga, M. Obesity and Cancer Risk: Emerging Biological Mechanisms and Perspectives. Metabolism 2019, 92, 121-135. [CrossRef]

46. Picon-Ruiz, M.; Morata-Tarifa, C.; Valle-Goffin, J.J.; Friedman, E.R.; Slingerland, J.M. Obesity and Adverse Breast Cancer Risk and Outcome: Mechanistic Insights and Strategies for Intervention. CA Cancer J. Clin. 2017, 67, 378-397. [CrossRef]

47. Burgess, S.; Davies, N.M.; Thompson, S.G.; on behalf of EPIC-InterAct Consortium. Instrumental Variable Analysis with a Nonlinear Exposure-Outcome Relationship. Epidemiology 2014, 25, 877-885. [CrossRef] [PubMed] 